\title{
Magnetic-field-induced Luttinger insulator state in quasi-one-dimensional conductors
}

\author{
Victor M. Yakovenko and Anatoley T. Zheleznyak \\ cond-mat/9807061, July 3, 1998
}

\begin{abstract}
Department of Physics and Center for Superconductivity Research, University of Maryland, College Park, MD
\end{abstract} 20742, USA

\begin{abstract}
We present a heuristic, semiphenomenological model of the anomalous temperature $(T)$ dependence of resistivity $\rho_{x x}$ recently observed experimentally in the quasi-one-dimensional (Q1D) organic conductors of the (TMTSF) $)_{2} \mathrm{X}$ family in moderately strong magnetic fields. We suggest that a Q1D conductor behaves like an insulator $\left(d \rho_{x x} / d T<\right.$ $0)$, when its effective dimensionality is one, and like a metal $\left(d \rho_{x x} / d T>0\right)$, when its effective dimensionality is greater than one. Applying a magnetic field reduces the effective dimensionality of the system and switches the temperature dependence of resistivity between the insulating and metallic laws depending on the magnitude of the magnetic field and its orientation along the "magic" and "nonmagic" angles.

Keywords: Many-body and quasiparticle theories; Transport measurements, conductivity, Hall effect, magnetotransport; Organic conductors based on radical cation and/or anion salts; Organic superconductors.
\end{abstract}

In recent experiments [1, 2, 3, a very unusual temperature $(T)$ dependence of the longitudinal resistivity $\rho_{a a}$ was observed in quasi-one-dimensional (Q1D) organic conductors (TMTSF) ${ }_{2} \mathrm{ClO}_{4}$ (at the ambient pressure) and (TMTSF) ${ }_{2} \mathrm{PF}_{6}$ (at a pressure about $9 \mathrm{kbar}$ ) in a moderately strong magnetic field $H$ of the order of $10 \mathrm{~T}$ applied along the $\mathbf{c}$ axis. At high temperatures, $\rho_{a a}$ decreases with decreasing temperature, as in conventional metals. However, below a certain magnetic-field-dependent temperature $T_{\min } \sim 20 \mathrm{~K}$, resistivity starts to grow with decreasing temperature: $d \rho_{a a} / d T<0$ at $T<T_{\min }$. In other words, the behavior of the system changes from metallic, $d \rho_{a a} / d T>0$, to insulating, $d \rho_{a a} / d T<0$, when the temperature is below $T_{\min }$. The temperature $T_{\min }$ increases with the increase of magnetic field. As the temperature is lowered further, $\rho_{a a}(T)$ continues to grow until another temperature scale $T_{\max } \sim 8 \mathrm{~K}<T_{\min }$ is reached. At $T<T_{\max }$, the behavior of the system starts to depend crucially on the exact orientation of the magnetic field [3]. If the magnetic field is tilted to a Lebed's "magic angle" 四, i.e. lies in a plane formed by the direction a of the chains and another integer crystallographic direction, such as $\mathbf{c}$ or $\mathbf{c}+\mathbf{b}, \rho_{a a}$ recovers the metallic behavior $d \rho_{a a} / d T>0$ at $T<T_{\max }$. For other, generic orientations of the magnetic field, $\rho_{a a}$ retains the nonmetallic behavior, either continuing to grow with decreasing temperature: $d \rho_{a a} / d T<0$, or saturating at a high constant value. The temperature $T_{\max }$ does not depend appreciably on the magnetic field. If at $T<T_{\max }$ the magnetic field is rotated in the plane perpendicular to the direction a of the chains, $\rho_{a a}$ exhibits sharp minima at the "magic angles".

This behavior of resistivity completely contradicts the conventional Fermi-liquid picture of a metal with an open Fermi surface. We suggest that the following theoretical picture [5] may qualitatively account for the unusual behavior of (TMTSF) ${ }_{2} \mathrm{PF}_{6}$.

The orbital effect of a c-axis magnetic field $H$ on a Q1D conductor is characterized by the cyclotron energy $E_{H}=e b H v_{F} / C$, where $e$ is the electron charge, $b$ is the distance between the chains in the $\mathbf{b}$ direction, $v_{F}$ is the Fermi velocity, and $C$ is the speed of light. We estimate that $E_{H} / H \approx 1.8 \mathrm{~K} / \mathrm{T}$, so the magnitude of the cyclotron energy, $E_{H} \approx 14 \mathrm{~K}$ at $H=7.8 \mathrm{~T}$, is close to the temperature of the resistivity minimum at that magnetic field, $T_{\min } \sim 20 \mathrm{~K}$. Taking into account that the minimum of resistivity clearly has a magnetic origin (it does not exist without magnetic field), and $T_{\min }$ grows with the increase of magnetic field, we suggest that the minimum of resistivity occurs when the temperature reaches the energy scale of the magnetic field; that is, $T_{\min } \approx E_{H}$.

Now we need to identify the nature of the second energy scale in the problem, the temperature of the resistivity maximum, $T_{\max }$. At the temperatures $T>T_{\max }$, it appears that $\rho_{a a}$ depends only on the magnetic field projection on the $\mathbf{c}^{*}$ axis perpendicular to the $\mathbf{a}$ and b directions. From this observation, we may conclude that at $T>T_{\max }$ the system behaves effectively as a two-dimensional (2D) system; that is, the coupling between the chains in the $\mathbf{c}$ direction is not relevant. On the other hand, at $T<T_{\max }$ the coupling along the $\mathbf{c}$ axis becomes important. This is manifested by the magic angles effect, which is an essentially threedimensional (3D) phenomenon involving both the $\mathbf{b}$ and c axes. The coupling between the chains along the $\mathbf{c}$ axis is characterized by the electron tunneling amplitude $t_{c}$, whose magnitude is believed to be of the order of $10 \mathrm{~K}$, which is close to $T_{\max } \sim 8 \mathrm{~K}$. Thus, we 
suggest that the electron tunneling amplitude $t_{c}$ sets the temperature scale $T_{\max }$ of the resistivity maximum: $T_{\max } \approx t_{c}$. This conjecture is supported by the experimental fact that $T_{\max }$ (unlike $T_{\min }$ ) does not depend appreciably on the magnetic field [2, 3].

Taking into account these energy scales, we identify three qualitatively different regimes in the behavior of a Q1D system in a magnetic field:

1) High temperatures: $E_{H} \approx T_{\min }<T<t_{b}$. In this region, the temperature is greater than both the magnetic energy $E_{H}$ and the electron tunneling amplitude $t_{c}$ along the c axis, but lower than the tunneling amplitude $t_{b}$ along the $\mathbf{b}$ axis. Thus, we may neglect both the magnetic field and the coupling between the chains along the $\mathbf{c}$ axis and treat the system as a normal 2D Fermi liquid without magnetic field. This results in the quadratic law $\rho_{a a} \sim T^{2}$ and the metallic behavior of the resistivity $d \rho_{a a} / d T>0$.

2) Intermediate temperatures: $t_{c} \approx T_{\max }<T<$ $T_{\min } \approx E_{H}$. In this region, the temperature is still greater than the coupling between the chains along the c axis, so the system remains 2D; however, the effect of the magnetic field becomes important. It is known that, in the presence of a magnetic field along the c axis, the motion of electrons along the $\mathbf{b}$ axis becomes quantized, and the dispersion law of electrons becomes one-dimensional (1D) [6]. Even though the spectrum of electrons becomes 1D and their wave functions become localized in the $\mathbf{b}$ direction, the wave functions still spread over many chains (if $E_{H} \ll t_{b}$ ), which results in a considerable interaction between different chains. So the system is not truly 1D, because it does not consist of completely decoupled 1D chains. Nevertheless, we may expect that, at least, some $1 \mathrm{D}$ features would be present in this regime and, via a mechanism that need to be identified, would lead to an insulating transport behavior $d \rho_{a a} / d T<0$. The conjecture that the insulating behavior is caused by the magnetic-field-enforced "one-dimerization" requires detailed studying of a specific mechanism.

3) Low temperatures: $T<T_{\max } \approx t_{c}$. In this region, the coupling between the $(\mathbf{a}, \mathbf{b})$ planes becomes important. The magnetic field pointing exactly along the $\mathbf{c}$ axis does not affect the electron motion along that axis. Thus, in addition to the magnetic-field-enforced 1D dispersion law discussed in part 2), the system acquired an extra dispersion in the c direction and becomes effectively 2D, which results in a metallic, Fermiliquid behavior $d \rho_{a a} / d T>0$. If the magnetic field does not point along the $\mathbf{c}$ axis, the component of the field perpendicular to the c axis suppresses the energy dispersion along that axis, so the system remains effectively $1 \mathrm{D}$ and insulating: $d \rho_{a a} / d T<0$. If the direction of the field is close to the $\mathbf{c}$ axis, we expect resistivity to decrease with decreasing temperature in the range
$E_{H}^{(c)}<T<T_{\max } \approx t_{c}$ and start increasing again at $T<E_{H}^{(c)}$, where $E_{H}^{(c)}$ is the cyclotron energy of the electron motion along the $\mathbf{c}$ axis, which is proportional to the projection of the magnetic field perpendicular to the $\mathbf{c}$ axis. The same arguments apply not only to the $\mathbf{c}$ axis, but also to the $\mathbf{c}+\mathbf{b}$ axis and other integer crystallographic directions $m \mathbf{c}+n \mathbf{b}$. However, because the electron tunneling amplitudes in these directions decrease rapidly with the increase of the integers $m$ and $n$, the effect is clearly visible experimentally only for the $\mathbf{c}+\mathbf{b}$ axis.

In summary, we suggest that the unusual transport behavior of (TMTSF $)_{2} \mathrm{PF}_{6}$ results from the changes in the effective dimensionality of the system caused by the applied magnetic field. The system is $2 \mathrm{D}$ at $E_{H}<T<t_{b}$ and effectively $1 \mathrm{D}$ at $t_{c}<T<E_{H}$. At $T<t_{c}$ the system is effectively $2 \mathrm{D}$ for the magic orientations of the magnetic field and effectively 1D for generic orientations. Whenever the system is $2 \mathrm{D}$ (or 3D), it is a normal Fermi liquid, and the temperature dependence of resistivity is metallic. Whenever the system is effectively $1 \mathrm{D}$, the temperature dependence of resistivity is insulating. The latter state of the system might be called the magnetic-field-induced Luttinger insulator (MFILI), by analogy with the term "Luttinger liquid", which refers to the metallic state of a $1 \mathrm{D}$ system.

In Ref. [5], we critically analyzed whether various microscopic models suggested in literature can produce such a behavior and found that none of the models is fully satisfactory. In particular, we performed detailed analytical and numerical calculations within the magnetic-field-induced spin-density-wave precursor scenario [7] and found that the theoretical results do not agree with the experiment. Nevertheless, we can predict some experimental effects based on our heuristic picture. In Refs. [8, 9], oscillations of the transverse resistivity $\rho_{c c}$ upon rotation of a magnetic field in the $(\mathbf{a}, \mathbf{c})$ plane were observed. It was found that a small magnetic field along the $\mathbf{b}$ axis destroys the oscillations [9]. We predict that, if a magnetic field is rotated in the magic plane from the $\mathbf{b}+\mathbf{c}$ direction toward the $\mathbf{a}$ direction, Danner's oscillations should exist, even though the magnetic field has a finite $\mathbf{b}$-component. The suggested geometry has an advantage over the geometry of the experiment [9], where the magnetic field had a fixed b-component, that Danner's oscillations would not be mixed up with Lebed's oscillations occurring when the magnetic field is rotated in the $(\mathbf{b}, \mathbf{c})$ plane. Our prediction is based on the idea that Danner's oscillations require that the electron motion in the third direction is not suppressed by the magnetic field, which happens only when the magnetic field belongs to a magic plane. A two-axes rotation of the magnetic field was performed in Ref. [10, but with a different the choice of geome- 
try. We also predict that Danner's oscillations should disappear at $T>T_{\max }$, where the electron dispersion in the third direction is smeared out by temperature.

\section{References}

[1] K. Behnia et al., Phys. Rev. Lett. 74, 5272 (1995).

[2] D. Jérome, in Correlated Fermions and Transport in Mesoscopic Systems, edited by T. Martin, G. Montambaux, and J. Trân Thanh Vân (Editions Frontieres, Gif-sur-Yvette, 1996), p. 95.

[3] E. I. Chashechkina and P. M. Chaikin, Phys. Rev. Lett. 80, 2181 (1998).

[4] J. Phys. (Paris) I 6, 1819 (1996).

[5] A. T. Zheleznyak and V. M. Yakovenko, condmat/9802172.

[6] L. P. Gor'kov and A. G. Lebed', J. Phys. Lett. (Paris) 45, L433 (1984).

[7] L. P. Gor'kov, Europhys. Lett. 31, 49 (1995); J. Phys. (Paris) I 6, 1697 (1996).

[8] G. M. Danner, W. Kang, and P. M. Chaikin, Phys. Rev. Lett. 72, 3714 (1994).

[9] G. M. Danner and P. M. Chaikin, Phys. Rev. Lett. 75, 4690 (1995).

[10] I. J. Lee and M. J. Naughton, Phys. Rev. B 57, 7423 (1998). 